and local turbulence in the EIA, combined with strong thigh flexion and an abnormally sinuous section of artery, may be the cause of a slow progressive stenotic intimal thickening. The high incidence of falls or crashes at competition level cycling has not previously been considered as a predisposing factor.

This case was atypical in that complete rather than partial occlusion had occurred, so that the patient had begun to feel symptoms during normal activity and not just maximal exercise.

It appears from the pathological findings that, despite the transient nature of the early symptoms, trauma may have been a factor in the development of the symptoms. As far as we are aware this is the first report of ischaemia in an athlete caused by trauma connected with cycling.

Despite its rarity, awareness of this condition is essential to prevent delays in diagnosis. Our case illustrates the importance of comprehensive evaluation of athletes. Vascular pathology must be considered when assessing patients complaining of vague unilateral leg pains during or after competition. Cyclists in particular may well be predisposed to stenosis or occlusion of the EIA.

1 Walder J, Mosimann F, Van Melle G, Mosimann R. A propos de l'endofibrose iliaque chez deux courers cyclists. Helv Chir Acta 1984;51:793-5.

2 Mosimann R, Walder J, Van Melle G. Stenotic intimal thickening of the external iliac artery. Illness of competition cyclists. Vascular Surgery 1985;19:258-63.

3 Chevalier JM, Enon B, Walder J, Barral X, Pillet J, Megret $A$, et al. L'endofibrose iliaque externe du cyclist du competition. Une pathologie arterielle meconnue. Annales de Chirurgie Vasculaire 1986;1:297-303.

4 Rousselet MC, Saint-Andre JP, L'Hoste P, Enon B, Megret A, Chevalier JM. Stenotic intimal thickening of the externa iliac artery in competition cyclists. Hum Pathol 1990;21 524-9.
Department of University Hospital of Wales, Heath Park, Cardiff CF4 4XW, United Kingdom I A Wright

N D Pugh

\section{Department of} Cardiology, University Hospital of Wales, Heath Park, Cardiff CF4 4XW, United Kingdom J Goodfellow A G Fraser

Department of Radiology, University Hospital of Wales, Heath Park, Cardiff CF4 4XW, United Kingdom

A M Wood

Department of Surgery, University Hospital of Wales, Heath Park, Cardifi CF4 4XW, United Kingdom

I F Lane

Correspondence to: Miss I A Wright, Department of Medical Physics and Bioengineering, University Hospital of Wales, Heath Park, Cardiff CF4 4XW, United Kingdom.

Accepted for publication 18 December 1996

\title{
Dynamic obstruction of the external iliac artery in endurance athletes and its relationship to endothelial function: the case of a long distance
} \section{runner}

\author{
Isabel A Wright, Neil D Pugh, Jonathan Goodfellow, Andrew M Wood, Ian F Lane, \\ Alan G Fraser
}

\begin{abstract}
There have been recent reports of exercise induced claudication in endurance trained athletes attributed to narrowing of the external iliac artery. Most patients have been competitive cyclists, and intimal hyperplasia has been cited as the cause. The case is reported here of a long distance runner who presented with similar symptoms.
\end{abstract}

(Br F Sports Med 1997;31:156-158)

Keywords: external iliac artery; long distance runner; diabetes mellitus

Since the early 1980 s there have been several reports documenting stenoses of the external iliac artery (EIA) in competitive cyclists. ${ }^{1-3}$ They describe how intimal hyperplasia/ endofibrosis produces a narrowing of the EIA, leading to lower limb claudication at maximal effort. It is thought that intimal hyperplasia may be produced in response to repetitive arterial injury. Endarterectomy and vein patch angioplasty have been used to treat some cases. $^{2}$
Here we describe a long distance runner who presented with similar symptoms, apparently caused by arterial spasm.

\section{Case report}

A 39 year old female marathon runner presented with an 18 month history of acute pain in her right thigh and calf after running approximately 200 yards. She was a normotensive insulin dependent diabetic (of 18 months) and an ex-smoker ( 20 per day) from 12 years previously. Clinical examination was normal and there was no family history of cardiovascular disease.

Magnetic resonance imaging showed no evidence of lumbar canal stenosis or popliteal entrapment.

Resting Doppler studies showed a normal ankle/brachial systolic pressure index (ABPI) of 1.00 on the right and 1.06 on the left, with triphasic ankle waveforms bilaterally. There were raised velocities in the right common femoral artery (CFA) with a diffuse narrowing of the EIA as compared with the left on Duplex scan; however, this was not haemodynamically significant. Otherwise the lower limb vasculature appeared entirely normal. 
Table 1 Results of Doppler studies performed after stressing the aortoiliac segments

\begin{tabular}{llll}
\hline & Pre-exercise & Post-exercise & Percentage change \\
\hline ABPI $^{\star}$ & 1.10 & 0.93 & -15 \\
Right & 1.13 & 1.20 & +6 \\
Left & & & \\
EIA diameter (mm) & 5.6 & 6.3 & +13 \\
$\quad$ Right & 6.6 & 8.7 & +32 \\
Left & & & +46 \\
EIA PSV $(\mathrm{m} / \mathrm{s})$ & 1.95 & 2.84 & -5 \\
$\quad$ Right & 1.06 & 1.01 & \\
Left & & & \\
\hline
\end{tabular}

$\star \mathrm{ABPI}=$ ankle/brachial systolic pressure index; EIA $=$ external iliac artery; $\mathrm{PSV}=$ peak systolic velocity.

Arteriography was performed, via a right CFA puncture. Initial runs showed a stenosis of the distal right EIA and proximal CFA, probably due to spasm. Intra-arterial nitrate was administered which produced a return to near normal appearances, apart from a very mild residual proximal CFA stenosis. Elsewhere the vasculature appeared entirely normal.

During this procedure, intravascular ultrasound assessment was performed with a 20 $\mathrm{MHz}$ transducer (Boston/Hewlett Packard). This demonstrated concentric thickening of the media along the length of the EIA, but the major finding was a large asymmetric thickening in the distal EIA. At this site the lumen diameter was comparable to other segments of the EIA, but the artery itself was dilated.

The aortoiliac segments were subsequently stressed by exercise. Resting ABPI, diameter, and peak systolic velocity (PSV) measurements were recorded (table 1). The patient was exercised until symptoms were induced and the Duplex measurements were repeated. A slight unilateral fall in ABPI was noted; however, the right EIA velocity increased by $46 \%$ compared with a negligible change on the left, and right EIA diameter increased by only $13 \%$ compared with a $32 \%$ increase on the left, as measured from the B-mode image. Extremely turbulent flow was evident in the right EIA.

Diabetes mellitus is known to be associated with endothelial dysfunction. ${ }^{4}$ Using high resolution ultrasonic vessel wall tracking we non-invasively assessed endothelial function by measuring flow mediated endothelium dependent dilatation in the brachial artery compared with the endothelium independent dilatation produced by sublingual glyceryl trinitrate as previously described. ${ }^{5}$ Endothelium dependent dilatation was attenuated ( $1.2 \%$ compared with $8.8 \%$ in normal subjects of similar age), whereas endothelium independent responses were preserved. This indicated the presence of systemic endothelial dysfunction.

The patient was prescribed a course of vasodilators (sustained release isosorbide mononitrate, sublingual glyceryl trinitrate) but this was discontinued because of headaches.

\section{Discussion}

Previous reports of EIA narrowing in competition cyclists cite intimal hyperplasia or endofibrosis as the cause. ${ }^{1-3}$ Arterial fibromuscular dysplasia is a non-atherosclerotic vascular disease which falls into three main categories: intimal fibroplasia/hyperplasia, medial fibromuscular dysplasia, and periarterial/ periadventitial fibroplasia. ${ }^{6}$ Intimal fibroplasia and medial hyperplasia, in addition to being indistinguishable radiologically, are often mistaken for atherosclerotic lesions angiographically. In our case, on intravascular ultrasound scanning, the intima appeared to be preserved and the lesion appeared to lie within the media, although without histopathology the exact nature of this lesion cannot be identified unequivocally. However, it would appear that this lesion may be caused by either medial hyperplasia or early atherosclerosis. Irrespective of the nature of the lesion, it was reported angiographically as being mild, at rest, and the Duplex scan and ABPI indicate that it was not haemodynamically significant. Elsewhere, both angiographically and on Duplex scan, the lower limb vasculature appeared entirely normal. It is unlikely therefore that this stenosis or indeed a stenosis of up to $30 \%$ reported by Rousselet et $a l^{1}$ would be large enough alone to produce a tight functional stenosis even at maximal effort where the patient's cardiac output may be of the order of 30 litres $/ \mathrm{min}^{7}$

Interestingly in this case we have found evidence of significant endothelial dysfunction. Endothelial dysfunction characterised by impaired NO release is increasingly recognised as an early and important feature of vascular disease. ${ }^{8}$ Flow mediated dilatation has been shown to be an endothelium dependent phenomenon mediated via release of NO in response to changes in shear stress. ${ }^{9}$ We have previously demonstrated that endothelial dysfunction manifested as impaired flow mediated dilatation is present in diabetics, ${ }^{10}$ which may explain the high incidence of vascular disease in these patients.

As expected, the patient in our study had evidence of systemic endothelial dysfunction with loss of flow mediated dilatation. Although her history favours the onset of atherosclerosis, the haemodynamic information and endothelial function tests suggest that the functional stenosis produced on exercise is a result of a combination of endothelial dysfunction and the presence of a fixed lesion producing inhibition of flow mediated vasodilatation. In the case of other endurance athletes exhibiting similar symptoms, it may be possible that endothelial dysfunction contributes significantly to the functional stenosis during exercise. In our case the cause of endothelial dysfunction is likely to be diabetes but in other athletes the aetiology may be related to localised endothelial dysfunction at the site of the lesion. The underlying phenomenon responsible may be insufficient arterial dilatation in response to increased flow, which may be due to either localised endothelial dysfunction as a result of intimal hyperplasia or localised rigidification of the arterial wall at the site of the lesion. Further work is needed in this area.

1 Rousselet M, Saint-Andre J, L'Hoste P, Enon B, Megret A Chevalier J. Stenotic intimal thickening of the external iliac artery in competition cyclists. Hum Pathol 1990;21:524-9. thickening of the external iliac artery: illness of the 
competition cyclists? Report of two cases. Vascular Surgery 1985;19:258-63.

3 Abraham P, Leftheriotis G, Bourre Y, Chevalier J, Saumet J. Echography of external iliac artery endofibrosis in cyclists. Am $\mathcal{F}$ Sports Med 1993;21:861-3.

4 Johnstone MT, Creager SJ, Scales KM, Cusco JA, Byron KL, Creager MA. Impaired endothelium-dependent vasodilation in patients with insulin dependent diabetes mellitus. Circulation 1993;88:2510-16.

5 Ramsey MW, Goodfellow J, Jones CJH, Luddington LA, Lewis MJ, Henderson AH. Endothelial control of arterial distensibilty is impaired in congestive heart failure. Circulation 1995;92:3212-19.

6 Luscher T, Lie J, Stanson A, Houser O, Hollier L, Sheps S.
Arterial fibromuscular dysplasia. Mayo Clin Proc 1987;62. 931-52.

7 Astrand P, Cuddy T, Saltin B, Stenberg J. Cardiac output during submaximal and maximal work. $\mathcal{f} A p p l$ Physio 1964;19:268-74

8 Griffith T. Modulation of blood flow and tissue perfusion by endothelium-derived relaxing factor. Exp Physiol 1994;79: 873-913.

9 Joannides R, Haefeli WE, Lilylinder VR, El Hassan B, Thuillez $C$, Luscher $T$. Nitric oxide is responsible for flow dependent dilatation of human peripheral conduit arteries in vivo. Circulation 1995;91:1314-19.

10 Goodfellow J, Ramsey M, Luddington LA, Jones CJH Owens DR, Lewis MJ, et al. Flow-related endothelial dilatation is impaired in non-insulin dependent diabetics. $B M^{9}$ t996;312:744-5.

\section{Commentary}

As a racing cyclist myself, I am only too aware of the frequency with which competitors land on their buttocks during competitive spills, particularly while riding in track events. Fortunately, these accidents usually only produce bruises, abrasions, and multiple splinters when wooden tracks are being used. It is indeed intriguing to think that there may be damage to the intima of the external iliac artery as a direct result of such trauma. It is fascinating to see a paper presenting a case of external iliac artery stenosis in a runner, albeit an insulin dependent diabetic competitor. As a clinician, I shall now consider more seriously the possibility of arterial causes when attempting to diagnose leg pain in competitors, particularly cyclists. Once again, these cases indicate the importance of taking a good clinical history and, where possible, assessing the athlete after exercise. 